POS PROCEEDINGS

\title{
CMSSM with Yukawa quasi-unification
}

\section{N. Karagiannakis ${ }^{*+}$}

Physics Division, School of Technology, Aristotle University of Thessaloniki, Thessaloniki 54124, Greece

E-mail: nikar@auth.gr

\section{G. Lazarides}

Physics Division, School of Technology, Aristotle University of Thessaloniki, Thessaloniki 54124, Greece

E-mail: lazadidedeng.auth.gr

\section{Pallis}

Department of Physics, University of Cyprus, P.O. Box 20537, CY-1678 Nicosia, CYPRUS

E-mail: kpalliseauth.gr

We present an updated analysis of the constrained minimal supersymmetric standard model with $\mu>0$ supplemented by an "asymptotic" Yukawa coupling quasi-unification condition, which allows an acceptable b-quark mass. Imposing constraints from the cold dark matter abundance in the universe, B physics, the muon anomalous magnetic moment, and the mass mh of the lightest neutral CP-even Higgs boson, we find that the lightest neutralino cannot act as a cold dark matter candidate. This is mainly because the upper bound on the lightest neutralino relic abundance from cold dark matter considerations, despite the fact that this abundance is drastically reduced by neutralino-stau coannihilations, is incompatible with the recent data on the branching ratio of $B_{s} \rightarrow \mu^{+} \mu^{-}$. Allowing for a different particle, such as the axino or the gravitino, to be the lightest supersymmetric particle and, thus, constitute the cold dark matter in the universe, we find that the predicted $\mathrm{mh}$ 's in our model favor the range (119 - 126) GeV.

Proceedings of the Corfu Summer Institute 2011 School and Workshops on Elementary Particle Physics and Gravity

September 4-18, 2011

Corfu, Greece

\footnotetext{
* Speaker.

${ }^{\dagger}$ Based on Talk presented at Corfu 2011 Workshop "CMSSM with Yukawa quasi-unification". (Prepared for the proceedings)

*We would like to thank A. Djouadi, N. Mahmoudi, K.A. Olive, P. Paradisi, Q. Shafi, and J. Wells for useful discussions. This work was supported by the European Union under the Marie Curie Initial Training Network 'UNILHC' PITN-GA-2009-237920, by the European Union (European Social Fund - ESF) and Greek national funds through the Operational Program "Education and Lifelong Learning" of the National Strategic Reference Framework (NSRF) Research Funding Program: Heracleitus II. Investing in knowledge society through the European Social Fund.
} 


\section{Introduction}

A highly predictive version of the Minimal Supersymmetric Standard Model (MSSM) with the addition of universal boundary conditions for soft supersymmetry (SUSY) braking parameters is the Constrained MSSM (CMSSM) [2, 3, 4] . In order this model to achieve 'asymptotic' Yukawa Unification (YU) [6], which means the exact unification of the third generation Yukawa coupling constants $h_{t}, h_{b}$ and $h_{\tau}$ respectively, we embed CMSSM in a SUSY grand unified theory (GUT) with a gauge group containing $S U(4)_{c}$ and $S U(2)_{R}$ [5]. The simplest GUT gauge group of this kind is the so called Pati-Salam (PS) group $G_{P S}=S U(4)_{c} \times S U(2)_{L} \times S U(2)_{R}[7,8]-$ for YU within $S O(10)$, see Refs $[9,10]$.

In the CMSSM, the masses of top-quark and tau-lepton, combined with YU, restrict the value of $\tan \beta \sim 50$. Considering the experimental values of the above masses, a serious problem rises; the mass $m_{b}$ of the b-quark become unacceptable for both signs of the parameter $\mu$. The cause of this, is the presence of sizable SUSY corrections [11] to $m_{b}$ (about 20\%), which arise [11, 12] from sbottom-gluino (mainly) and stop-chargino loops and have the sign of $\mu$ - with the standard sign convention of Ref. [13]. The predicted tree-level $m_{b}\left(M_{Z}\right)$, which turns out to be close to the upper edge of its 95\% confidence level (c.1.) experimental range, receives, for $\mu>0[\mu<0]$, large positive [negative] corrections which drive it well above [a little below] the allowed range. As a consequence, for both signs of $\mu$, YU leads to an unacceptable $m_{b}\left(M_{Z}\right)$ with the $\mu<0$ case being much less disfavored.

Usually, the resolution of this discrepancy comes with the introduction of several kinds of nonuniversalities in the scalar $[9,10]$ and/or gaugino $[15,14]$ sector of MSSM with an approximate preservation of YU. Instead of that, concrete SUSY GUT models based on the PS gauge group can be constructed [16] which naturally yield a moderate deviation of the b-quark mass for both signs of $\mu$ within the CMSSM. The key here is that the Higgs sector of the simplest PS model [7, 8] is extended so that the electroweak Higgs fields are not exclusively contained in a $S U(2)_{L} \times S U(2)_{R}$ bidoublet superfield but receive subdominant contributions from other representations too. As a consequence, a moderate violation of YU is naturally obtained, which can allow an acceptable b-quark mass even with universal boundary conditions. It is also remarkable that the resulting extended SUSY PS models support new successful versions [17] of hybrid inflation based solely on renormalizable superpotential terms.

These models provide us with a set of 'asymptotic' Yukawa quasi-unification conditions, which replace exact YU and can be applicable $[16,18,19]$ for both signs of the MSSM parameter $\mu$. We focus here on the $\mu>0$ case since $\mu<0$ is strongly disfavored by the constraint arising from the deviation $\delta \alpha_{\mu}$ of the measured value of the muon anomalous magnetic moment $\alpha_{\mu}$ from its predicted value $\alpha_{\mu}^{S M}$ in the standard model (SM). Indeed, $\mu<0$ is defended only at $3 \sigma$ by the calculation of $\alpha_{\mu}^{S M}$ based on the $\tau$-decay data, which is presented in Ref. [20], whereas there is a stronger and stronger tendency at present to prefer the $e^{+} e^{-}$-annihilation data for the calculation of $\alpha_{\mu}^{S M}$, which favor the $\mu>0$ regime. Moreover, in Ref. [21], it was claimed that, after some improvements, the $\tau$-based result shifts considerably towards the $e^{+} e^{-}$-based one.

Let us recall that, in this case, the suitable 'asymptotic' Yukawa quasi-unification condition applied $[16,18,19]$ is

$$
h_{t}: h_{b}: h_{\tau}=|1+c|:|1-c|:|1+3 c|
$$


Here, $c$ is a real parameter with values lying in the range $0<c<1$. Keeping third generation masses fixed, in contrast with the old version of the CMSSM [2, 3, 4], we can determine the values of $\tan \beta$ and $c$, so that Eq. (1.1) is satisfied. $\tan \beta$ is not a free parameter, but can be restricted within our set-up, via Eq. (1.1) to relatively large values. After this procedure, we are left with only three free parameters, the universal soft SUSY breaking parameters defined at $M_{G U T}$ scale

$$
M_{1 / 2}, m_{0} \text { and } A_{0}
$$

i.e. the common gaugino mass, scalar mass and trilinear scalar coupling constant, respectively. The space spanned by the above parameters can be restricted by employing several experimental and cosmological requirements as in Refs. [16, 18, 19] and most recently in Refs. [22, 23]. In this talk, we review the results of Ref. [22] implementing the following improvements:

- We do not take into account the upper bound on $m_{\tilde{\chi}}$ implied by the lower bound on $\delta \alpha_{\mu}$ from the $\tau$-based calculation of Ref. [20] raising, thereby, the upper bound on $m_{\tilde{\chi}}$ from the muon anomalous magnetic moment - see Sec.2.

- We employ the recently released data on the branching ratio of $B_{s} \rightarrow \mu^{+} \mu^{-}$[24] and the mass $m_{h}$ of the lightest CP-even Higgs boson [25]. As a consequence, our predictions in Ref. [22] for $m_{h}$ and the role of the lightest neutralino as cold dark matter (CDM) particle have been significantly altered.

In Sec. 2 we present in detail all the cosmological and phenomenological requirements which we considered in our investigation. Also, in Sec. 3 we derive all the restrictions on the parameter space of our model. Finally, in Sec. 4 our conclusions are summarized.

\section{Cosmological and Phenomenological Constraints}

Our investigation consists of three basic parts :

1. We integrate the two-loop renormalization group equations (RGE) for the gauge and Yukawa coupling constants and the one-loop ones for the soft SUSY breaking parameters between $M_{G U T}$ and a common SUSY threshold $M_{S U S Y} \simeq\left(m_{\tilde{t}_{1}} m_{\tilde{t}_{2}}\right)^{1 / 2}\left(\tilde{t}_{1,2}\right.$ are the stop mass eigenstates) determined in consistency with the SUSY spectrum.

2. At $M_{S U S Y}$, we impose radiative electroweak symmetry breaking (REWSB), evaluate the SUSY spectrum employing the latest version of the publicly available calculator SOFTSUSY [26], and incorporate the SUSY corrections to the $b$ and $\tau$ mass [12]. The corrections to the $\tau$-lepton mass $m_{\tau}$ (almost $4 \%$ ) lead $[16,18]$ to a small decrease of $\tan \beta$. From $M_{S U S Y}$ to $M_{Z}$, the running of gauge and Yukawa coupling constants is continued using the SM RGE.

3. The parameter space of our model can be restricted by using a number of phenomenological and cosmological constraints. We calculate them using the latest version of the publicly available code micrOMEGAs [27].

Let us briefly discuss these requirements - for similar recent analyses, see Ref. [28] for the CMSSM or Refs. [14, 29] for the MSSM with YU. 
SM Fermion Masses : The masses of the fermions of the third generation play a crucial role in the determination of the evolution of the Yukawa coupling constants. For the b-quark mass, we adopt as an input parameter in our analysis the $\overline{\mathrm{MS}} b$-quark mass, which at $1 \sigma$ is [30]

$$
m_{b}\left(m_{b}\right)^{\overline{\mathrm{MS}}}=4.19_{-0.06}^{+0.18} \mathrm{GeV}
$$

We evolve this range up to $M_{Z}$ using the central value $\alpha_{s}\left(M_{Z}\right)=0.1184$ [30] of the strong fine structure constant at $M_{Z}$ and then converted to the $\overline{\mathrm{DR}}$ scheme in accordance with the analysis of Ref. [31]. We obtain, at $95 \%$ c.1.

$$
2.745 \lesssim m_{b}\left(M_{Z}\right) / \mathrm{GeV} \lesssim 3.13
$$

where the central value is $m_{b}\left(M_{Z}\right)=2.84 \mathrm{GeV}$. For the top-quark mass, we use the central pole mass $\left(M_{t}\right)$ as an input parameter [32]:

$$
M_{t}=173 \mathrm{GeV} \Rightarrow m_{t}\left(m_{t}\right)=164.6 \mathrm{GeV}
$$

where $m_{t}\left(m_{t}\right)$ is the running mass of the $\mathrm{t}$ quark. We also take the central value $m_{\tau}\left(M_{Z}\right)=$ $1.748 \mathrm{GeV}[31]$ of the $\overline{\mathrm{DR}}$ tau-lepton mass at $M_{Z}$.

Cold Dark Matter Considerations : According to the WMAP results [33], the 95\% c.l. range for the CDM abundance is

$$
\Omega_{\mathrm{CDM}} h^{2}=0.1126 \pm 0.0072
$$

In the context of the CMSSM, the lightest neutralino $\tilde{\chi}$ can be the lightest supersymmetric particle (LSP) and, thus, naturally arises as a CDM candidate. In this case, the requirement that the $\tilde{\chi}$ relic abundance $\Omega_{\tilde{\chi}} h^{2}$ does not exceed the $95 \%$ c.l. upper bound from Eq. (2.4), i.e.

$$
\Omega_{\tilde{\chi}} h^{2} \lesssim 0.12
$$

strongly restricts the parameter space of the CMSSM. This is because $\Omega \tilde{\chi} h^{2}$ increases, in general, with $m_{\tilde{\chi}}$ and so an upper bound on $m_{\tilde{\chi}}$ can be derived from Eq. (2.5). In order to calculate $\Omega_{\tilde{\chi}} h^{2}$, micrOMEGAs includes accurately thermally averaged exact tree-level cross sections of all the possible (co)annihilation processes [3, 34], treats poles [4, 16, 35] properly, and uses one-loop QCD and SUSY QCD corrections [11, 16,36] to the Higgs decay widths and couplings to fermions. It should, though, be noted that the restrictions induced by Eq. (2.5) can be evaded if we adopt one (or a combination) of the following scenarios:

- The cosmological evolution before Big Bang nucleosynthesis (BBN) deviates from the standard one $[37,38]$. Since $\tilde{\chi}$ within the CMSSM is essentially a pure bino, the scenario which fits better this case is the low reheat temperature scenario with the decoupling of $\tilde{\chi}$ occurring before reheating. This scenario, however, is disfavored since it requires a very low reheat temperature $\sim(1-5) \mathrm{GeV}$. We will, thus, assume that the decoupling of the neutralino from the cosmic fluid occurs during the conventional radiation dominated era.

- The lightest neutralino is not the LSP and, thus, the relic density of another SUSY particle [39], which is the LSP, is to account for $\Omega_{\mathrm{CDM}} h^{2}$. This particle could be the gravitino [40] 
or the axino [41, 42]. The case of gravitino is tightly restricted in the CMSSM due to the BBN constraints imposed during the decay of the lightest ordinary supersymmetric particle (LOSP) to it. On the other hand, axino CDM [42] is, in general, possible once its mass and the reheat temperature are chosen appropriately. In such a case, $\tilde{\chi}$ may play the role of the LOSP and can contribute to the non-thermal production of the LSP. In particular, its contribution to the relic density of the LSP is equal to $\Omega_{\tilde{\chi}} h^{2}$ times the ratio of the LSP mass to $m_{\tilde{\chi}}$ and, thus, $\Omega_{\tilde{\chi}} h^{2}$ 's exceeding the bound in Eq. (??) can be perfectly acceptable.

The Branching Ratio $\mathbf{B R}(b \rightarrow s \gamma)$ of $b \rightarrow s \gamma$ : The most recent experimental word average for $\mathrm{BR}(b \rightarrow s \gamma)$ is known [43] to be $(3.52 \pm 0.23 \pm 0.09) \times 10^{-4}$ and its updated SM prediction is $(3.15 \pm 0.23) \times 10^{-4}$ [44]. Combining in quadrature the experimental and theoretical errors involved, we obtain the following constraints on this branching ratio at $95 \%$ c.l.:

$$
2.84 \times 10^{-4} \lesssim \mathrm{BR}(b \rightarrow s \gamma) \lesssim 4.2 \times 10^{-4}
$$

The computation of $\mathrm{BR}(b \rightarrow s \gamma)$ in the mi crOMEGAs package is described analytically in Ref. [36]. It includes [45] next-to-leading order (NLO) QCD corrections to the charged Higgs boson $\left(H^{ \pm}\right)$ contribution, the $\tan \beta$ enhanced contributions, as well as resummed NLO SUSY QCD corrections. The $H^{ \pm}$contribution interferes constructively with the SM contribution, whereas the SUSY contribution interferes destructively with the other two contributions for $\mu>0$. The SM plus the $H^{ \pm}$ and SUSY contributions initially increases with $m_{\tilde{\chi}}$ and yields a lower bound on $m_{\tilde{\chi}}$ from the lower bound in Eq. (2.6). (For higher values of $m_{\tilde{\chi}}$, it starts mildly decreasing.)

The Branching Ratio $\mathbf{B R}\left(B_{s} \rightarrow \mu^{+} \mu^{-}\right)$of $B_{s} \rightarrow \mu^{+} \mu^{-}$: The rare decay occurs via $Z$ penguin and box diagrams in the SM and, thus, its branching ratio is highly suppressed. The SUSY contribution, though, originating $[46,47]$ from neutral Higgs bosons in chargino-, $H^{ \pm}-$, and $W^{ \pm_{-}}$ mediated penguins behaves as $\tan ^{6} \beta / m_{A}^{4}$ ( $m_{A}$ is the mass of the CP-odd Higgs boson $A$ ) and hence is particularly important for large $\tan \beta$ 's, especially after the new more stringent $95 \%$ c.l. upper bound

$$
\mathrm{BR}\left(B_{s} \rightarrow \mu^{+} \mu^{-}\right) \lesssim 1.08 \times 10^{-8}
$$

recently reported by CMS and LHCb [24]. This new bound significantly reduces the previous bound [48], which we had adopted in [22]. The bound in Eq. (2.7) implies a lower bound on $m_{\tilde{\chi}}$ since $B_{s} \rightarrow \mu^{+} \mu^{-}$decreases as $m_{\mathrm{LSP}}$ increases.

The Branching Ratio $\mathbf{B R}\left(B_{u} \rightarrow \tau \nu\right)$ of $B_{u} \rightarrow \tau \nu$ : The purely leptonic decay $B_{u} \rightarrow \tau \nu$ proceeds via $W^{ \pm}$- and $H^{ \pm}$-mediated annihilation processes. The SUSY contribution, contrary to the SM one, is not helicity suppressed and depends on the mass $m_{H^{ \pm}}$of the chared Higgs boson, since it behaves $[47,49]$ as $\tan ^{4} \beta / m_{H^{ \pm}}^{4}$. The ratio $B_{u} \rightarrow \tau \nu$ of the CMSSM to the SM branching ratio of $B_{u} \rightarrow \tau \nu$ increases with $m_{\tilde{\chi}} x$ and approaches unity. It is to be consistent with the following $95 \%$ c.l. range [43]:

$$
0.52 \lesssim B R\left(B_{u} \rightarrow \tau v\right) \lesssim 2.04
$$

A lower bound on $m_{\tilde{\chi}}$ can be derived from the lower bound in this ineguality. 
Muon Anomalous Magnetic Moment : The quantity $\delta a_{\mu}$, which is defined in Sec. 1 , can be attributed to SUSY contributions arising from chargino-sneutrino and neutralino-smuon loops. The relevant calculation is based on the formulas of Ref. [50]. The absolute value of the result decreases as $m_{\tilde{\chi}}$ increases and its sign is positive for $\mu>0$. On the other hand, the calculation of $a_{\mu}^{\mathrm{SM}}$ is not yet completely stabilized mainly because of the ambiguities in the calculation of the hadronic vacuumpolarization contribution. According to the evaluation of this contribution in Ref. [20], there is still a discrepancy between the findings based on the $e^{+} e^{-}$-annihilation data and the ones based on the $\tau$-decay data - however, in [21], it was claimed that this discrepancy can be considerably ameliorated. Taking into account the more reliable calculation based on the $e^{+} e^{-}$data and the experimental measurements [51] of $a_{\mu}$, we obtain the following 95\% c.l. range - cf. [52]:

$$
12.7 \times 10^{-10} \lesssim \delta \alpha_{\mu} \lesssim 44.7 \times 10^{-10}
$$

A lower [upper] bound on $m_{\tilde{\chi}}$ can be derived from the upper [lower] bound in Eq. (2.9). As it turns out, only the upper bound on $m_{\tilde{\chi}}$ is relevant in our case. Taking into account the aforementioned computational instabilities and the common practice [28], we consider this bound only as an optional constraint.

Collider Bounds : For our analysis, the only relevant collider bound is the 95\% c.l. LEP bound [53] on the lightest CP-even neutral Higgs boson mass

$$
m_{h} \gtrsim 114.4 \mathrm{GeV}
$$

which gives a lower bound on $m_{\tilde{\chi}}$. However, we should keep in mind that recent data from ATLAS and CMS [25] provide a $99 \%$ c.l. upper bound $m_{h} \lesssim 128 \mathrm{GeV}$ and a hint in favor of the range (125士 1) $\mathrm{GeV}$. Allowing for a theoretical error of $\pm 1.5 \mathrm{GeV}$ and adding in quadrature the experimental and theoretical uncertainties, we construct the $1 \sigma$ range of interest [54]:

$$
123.2 \lesssim m_{h} / \mathrm{GeV} \lesssim 126.8
$$

The calculation of $m_{h}$ in the package SOF TSUSY [26] includes the full one-loop SUSY corrections and some zero-momentum two-loop corrections [55]. The results are well tested [56] against other spectrum calculators.

\section{Restrictions on the SUSY Parameters}

Imposing the requirements described above, we can delineate the allowed parameter space of our model. Throughout our investigation, we consider the central values for the SM parameters $M_{t}, m_{b}\left(M_{Z}\right), m_{\tau}\left(M_{Z}\right)$, and $\alpha_{s}\left(M_{Z}\right)$. We adopt the following conventions for the various lines and regions in the relevant figures (Figs. 2 and 3) - see Fig. 1:

- On the solid black line, Eq. (2.5) is saturated.

- The horizontally hatched region is allowed by Eq. (2.5).

- In the light gray region, the lightest stau $\tilde{\tau}_{2}$ is lighter than $\tilde{\chi}$. 


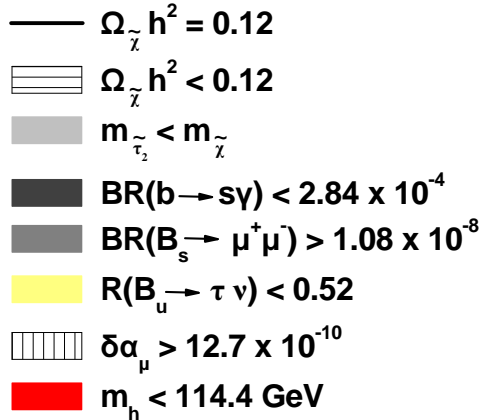

Figure 1: Summary of the conventions adopted in Figs. 2 and 3 for the various restrictions on the model parameters.

- The dark gray region is excluded by Eq. (2.6).

- The gray region is excluded by Eq. (2.7).

- The yellow region is excluded by the lower bound in Eq. (2.8).

- The vertically hatched region is favored by the lower bound in Eq. (2.9).

- The red region is excluded by Eq. (2.10).

Note that the upper bounds in Eqs. (2.6), (2.8), and (2.9) do not restrict the parameters of our model. The region with $\tilde{\tau}_{2}$ lighter than $\tilde{\chi}$ can not be excluded if the LSP is a neutral sparticle other than $\tilde{\chi}$. One should, though, make sure that the decay of $\tilde{\tau}_{2}$ to the LSP does not destroy the predictions of the standard BBN [40, 41].

We present the restrictions from all the requirements imposed in the $M_{1 / 2}-m_{0}$ plane for $A_{0} / M_{1 / 2}=0,1,-1$, and -2 in Fig. 2. From the relevant data, we observe that the lower bound in Eq. (2.8) is fulfilled for the mass of the CP-odd Higgs boson $m_{A} \gtrsim 520 \mathrm{GeV}$ and almost independently of the other parameters. Note also that, for $A_{0} / M_{1 / 2}=-1$ and -2 , the bound in Eq. (2.10) is violated for $M_{1 / 2}<400 \mathrm{GeV}$ and, consequently, does not appear in the relevant diagrams. It is obvious that, for all the $A_{0} / M_{1 / 2}$ 's considered in Fig. 2, we are left with no region allowed by all the restrictions of Sec. 2. This is due to the fact that the constraint in Eq. (2.5), which necessarily holds if $\tilde{\chi}$ is the LSP, is nowhere fulfilled simultaneously with the bound in Eq. 2.7. Note, finally, that the upper bound on $M_{1 / 2}$ from the lower bound in Eq. (2.9) is also nowhere satisfied simultaneously with the bound in Eq. (2.7) for the values taken for $A_{0} / M_{1 / 2}$ in Fig. 2. However, this conflict is less serious since, as we already explained in Sec. 2, the lower bound in Eq. (2.9) is considered here only as an optional constraint.

The constraint in Eq. (2.5) is, in general, satisfied in two well-defined distinct regions in the diagrams of Fig. 2, which are:

- The region to the left of the almost vertical part of the line corresponding to the upper bound on $M_{1 / 2}$ from Eq. (2.5), where the neutralino annihilation via the $s$-channel exchange of a $\mathrm{CP}$-odd Higgs boson $A$ is by far the dominant (co)annihilation process. However, this region is excluded by the constraints in Eqs. (2.7) and (2.8). On the other hand, it is well known see e.g. Refs. $[4,16]$ - that this region is extremely sensitive to variations of $m_{b}\left(M_{Z}\right)$. Indeed, we find that, as $m_{b}\left(M_{Z}\right)$ decreases, the $A$-boson mass $m_{A}$ increases and approaches $2 m_{\tilde{\chi}}$. The 

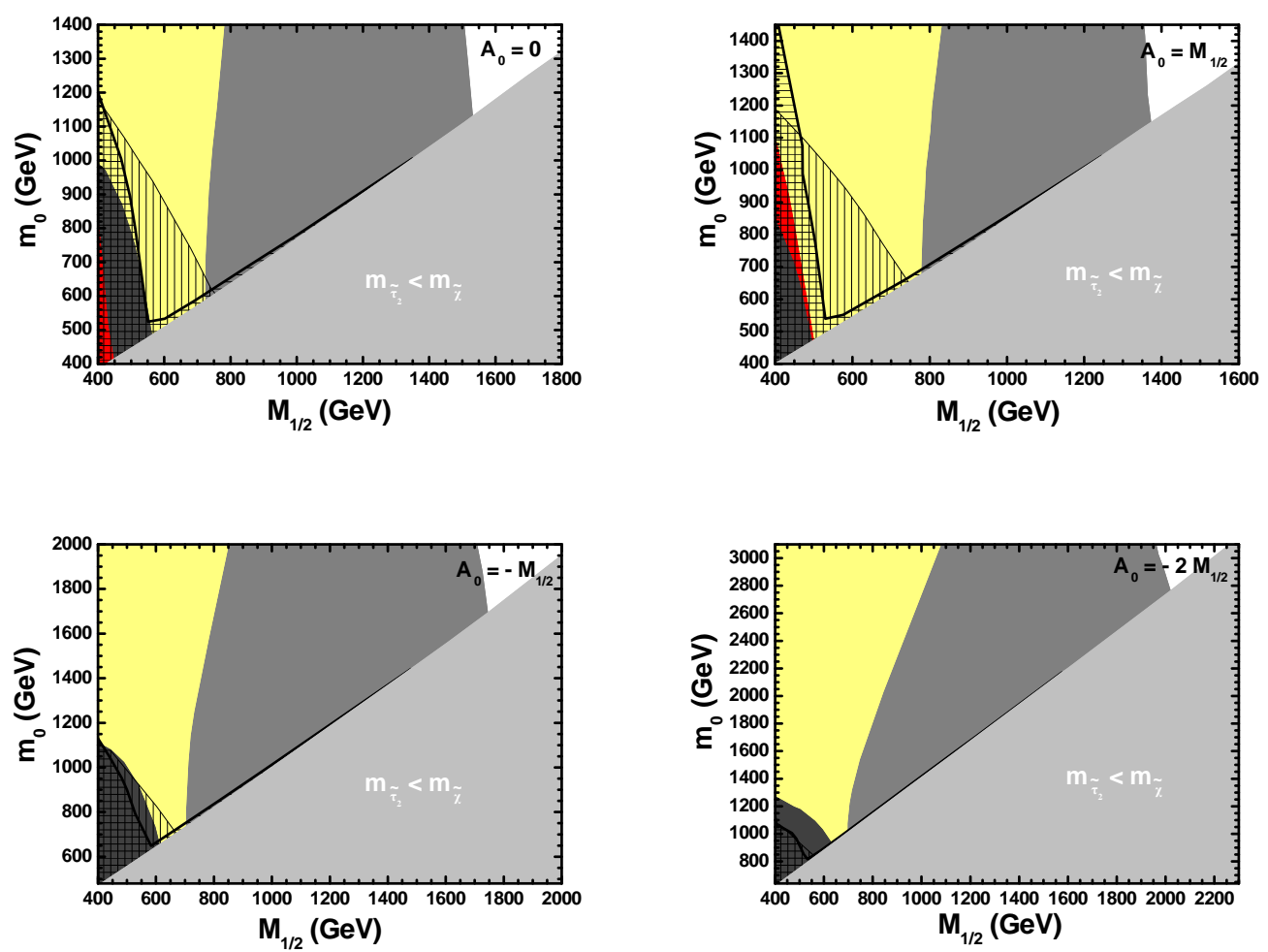

Figure 2: The restrictions in the $M_{1 / 2}-m_{0}$ plane for various values of $A_{0} / M_{1 / 2}$ indicated in the graphs. The conventions adopted are described in Fig. 1.

$A$-pole neutralino annihilation is then enhanced and $\Omega_{\tilde{\chi}} h^{2}$ is drastically reduced causing an increase of the upper bound on $M_{1 / 2}$. However, even if we reduce $m_{b}\left(M_{Z}\right)$, we do not find any $A$-pole neutralino annihilation region which is allowed by the requirements in Eqs. (2.7) and (2.8).

- The narrow region which lies just above the light gray area with $\tilde{\tau}_{2}$ lighter than the neutralino, where bino-stau coannihilations $[3,34]$ take over leading to a very pronounced reduction of $\Omega_{\tilde{\chi}} h^{2}$. A large portion of this region survives after the application of all the other requirements of Sec. 2 except for that in Eq. (2.7). To get a better understanding of this region, we can replace the parameter $m_{0}$ by the relative mass splitting $\Delta_{\tilde{\tau}_{2}}=\left(m_{\tilde{\tau}_{2}}-m_{\tilde{\chi}}\right) / m_{\tilde{\chi}}$ between $\tilde{\chi}$ and the lightest stau, which controls the strength of bino-stau coannihilations. The coannihilation region then approximately corresponds to $\Delta_{\tilde{\tau}_{2}}=0-0.25$. It is evident from Fig. 2 that the slope of the boundary line with $\Delta_{\tilde{\tau}_{2}}=0$ increases as $A_{0} / M_{1 / 2}$ moves away from zero in both directions. Note that this slope in our model turns out to be larger than the one obtained in other versions of the CMSSM - cf. [3] - with lower values of $\tan \beta$. As a consequence, small variations of $m_{0}$ or $M_{1 / 2}$ lead, in our model, to more drastic variations in $\Delta_{\tilde{\tau}_{2}}$.

To investigate further whether the incompatibility between the constraints in Eqs. (2.5) and (2.7) extends to all possible $A_{0} / M_{1 / 2}$ 's, we focus on the coannihilation regime and construct the regions allowed by all the restrictions of Sec. 2 in the $M_{1 / 2}-A_{0} / M 1 / 2$ plane for $\Delta_{\tilde{\tau}_{2}}=0$. We 


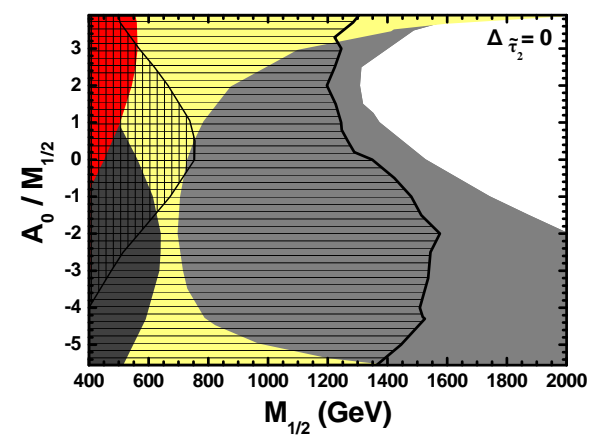

Figure 3: The restrictions in the $M_{1 / 2}-$ $A_{0} / M_{1 / 2}$ plane for $\Delta_{\tilde{\tau}_{2}}=0$ following the conventions of Fig. 1.

depict our results in Fig. 3. The choice $\Delta_{\tilde{\tau}_{2}}=0$ ensures the maximal possible reduction of $\Omega \tilde{\chi} h^{2}$ due to the $\tilde{\chi}-\tilde{\tau}_{2}$ coannihilation. So, for a given value of $A_{0} / M_{1 / 2}$, the maximal $M_{1 / 2}$ or $m_{\tilde{\chi}}$ allowed by Eq. (2.5), which holds under the assumption that the neutralino is the LSP, corresponds to $\Delta_{\tilde{\tau}_{2}}=0$. We find that, for $A_{0} / M_{1 / 2}<0$, processes with $\tilde{\tau}_{2} \tilde{\tau}_{2}^{*}$ in the initial state and $W^{ \pm} W^{\mp}$, $W^{ \pm} H^{\mp}$ in the final one become more efficient (with a total contribution to the effective cross section of about 14 to $22 \%$ as $A_{0} / M_{1 / 2}$ decreases from 0 to -2 ) and so coannihilation is strengthened and $m_{\tilde{\chi}}$ 's larger than in the $A_{0} / M_{1 / 2}>0$ case are allowed by Eq. (2.5). The overall maximal $M_{1 / 2} \simeq 1575 \mathrm{GeV}$ or $m_{\tilde{\chi}} \simeq 722 \mathrm{GeV}$ allowed by Eq. (2.5) is encountered at $A_{0} / M_{1 / 2} \simeq-2$ yielding $B_{s} \rightarrow \mu^{+} \mu^{-}=1.82 \times 10^{-8}$. Comparing the above upper bound on $M_{1 / 2}$ with the corresponding one in Fig. 3 of [22] (represented by a solid and two dotted black lines), we observe that here the bound is considerably enhanced in the region of low as well as the region of large values of $A_{0} / M_{1 / 2}$ since we do not consider the constraint from the lower bound on $\delta a_{\mu}$ from the $\tau$ based calculation. However, it always remains smaller than the lower bound on $M_{1 / 2}$ derived from Eq. (2.7) - note that for $3.7 \lesssim A_{0} / M_{1 / 2} \lesssim 3.9$ the overall lower bound on $M_{1 / 2}$ is derived from Eq. (2.8). Indeed, the smallest lower bound on $M_{1 / 2}=1306 \mathrm{GeV}$ or $m_{\tilde{\chi}} \simeq 590 \mathrm{GeV}$ is found at $A_{0} / M_{1 / 2} \simeq 2$ yielding $\Omega_{\tilde{\chi}} h^{2}=0.15$. Note that increasing $\Delta_{\tilde{\tau}_{2}}$ within the range $0-0.25$ does not alter the boundaries of the various constraints in any essential way, except the solid line which is displaced to the left shrinking, thereby, the area allowed by Eq. (2.5) considerably. Needless to say that the more stringent optional upper bound on $M_{1 / 2}$ from the lower bound in Eq. (2.9) is also not compatible with the constraint in Eq. (2.7). Consequently, for every $A_{0}$, there is no range of parameters simultaneously allowed by all the constraints and, therefore, $\tilde{\chi}$ can be now excluded as a CDM particle in our model.

The exclusion, in our model, of $\tilde{\chi}$ as a CDM candidate, resulting from the incompatibility between Eqs (2.5) and (2.7), is further strengthened if one tries to reconcile Eqs. (2.5) and (2.11). Indeed, the tension between the neutralino CDM and the new data on $m_{h}$ is quite generic within the CMSSM since the fulfillment of Eq. (2.11) requires a very heavy SUSY spectrum, which leads to conflict with Eq. $(2.5)$ - cf. Refs. [54, 57]. It would be interesting to investigate this issue in our model, which yields large values of $\tan \beta$ and so Eq. (2.11) can be possibly satisfied with a lighter SUSY spectrum relative to other versions of the CMSSM with lower $\tan \beta$ 's. Our results are presented in Fig. 4 , where we draw $m_{h}$ (bold lines) versus $m_{\tilde{\chi}}$ for $\Delta_{\tilde{\tau}_{2}} \simeq 0$ and $A_{0} / M_{1 / 2}=-2,-1$, 0, 1, and 3.9. In the table included in Fig. 4 , we also list the minimal $m_{\tilde{\chi}}$ 's, $\left.m_{\tilde{\chi}}\right|_{\min }$, for which the inequality in Eq. (2.7) and the lower bound in Eq. (2.8) are satisfied for given values of $A_{0} / M_{1 / 2}$ as well as the corresponding ranges of $\Omega_{\tilde{\chi}} h^{2}$ as $m_{\tilde{\chi}}$ varies from $\left.m_{\tilde{\chi}}\right|_{\min }$ to about $1 \mathrm{TeV}$. Since we 


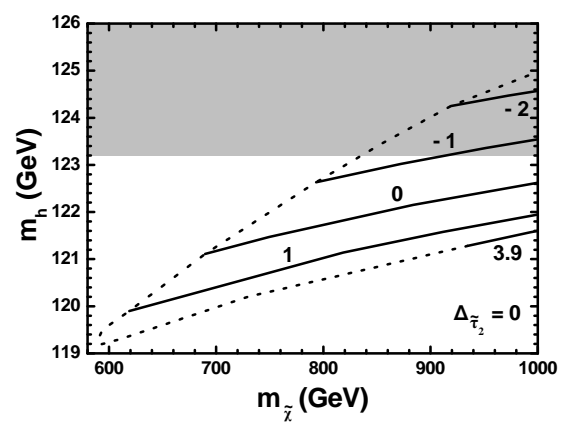

\begin{tabular}{ccc}
\hline$A_{0} / M_{1 / 2}$ & $\left.m_{\tilde{\chi}}\right|_{\min }$ & $\Omega_{\tilde{\chi}} h^{2}$ \\
\hline-2 & $919 \mathrm{GeV}$ & $0.22-0.32$ \\
-1 & $793 \mathrm{GeV}$ & $0.17-0.31$ \\
0 & $688 \mathrm{GeV}$ & $0.15-0.38$ \\
1 & $619 \mathrm{GeV}$ & $0.16-0.44$ \\
3.9 & $935 \mathrm{GeV}$ & $0.31-0.34$ \\
\hline
\end{tabular}

Figure 4: The variation of $m_{h}$ as a function of $m_{\tilde{\chi}}$ for $\Delta_{\tilde{\tau}_{2}} \simeq 0$ and various $A_{0} / M_{1 / 2}$ 's indicated on the curves and in the table included in this figure. In this table, listed are also the minimal $m_{\tilde{\chi}}$ 's for which the inequality in Eq. (2.7) and the lower bound in Eq. (2.8) are satisfied and the corresponding ranges of $\Omega_{\tilde{\chi}} h^{2}$ as $m_{\tilde{\chi}}$ increases from $\left.m_{\tilde{\chi}}\right|_{\min }$ to about $1 \mathrm{TeV}$. The minimal $m_{h}$ for all the values of $A_{0} / M_{1 / 2}$ is also depicted by a dotted line. The part of the region of Eq. (2.11) preferred by the recent LHC data which lies in the panel is painted light gray.

take $\Delta_{\tilde{\tau}_{2}} \simeq 0$, the derived $\Omega_{\tilde{\chi}} h^{2}$ takes its minimal possible value. In this plot, we also depict by a dotted line the value of $\left.m_{\tilde{\chi}}\right|_{\text {min }}$ for all possible $A_{0} / M_{1 / 2}$ 's and $\Delta_{\tilde{\tau}_{2}} \simeq 0$. This line terminates at $A_{0} / M_{1 / 2} \simeq 3.9$ since beyond this value the stability of the electroweak vacuum fails. The overall minimal $m_{h} \simeq 119 \mathrm{GeV}$ is encountered at $A_{0} / M_{1 / 2} \simeq 2$ and $m_{\tilde{\chi}} \simeq 590 \mathrm{GeV}$. It is interesting to note that $m_{h}$ increases with $m_{\tilde{\chi}}$ (or $M_{1 / 2}$ ) and as $A_{0}$ decreases and, eventually, this mass enters inside the gray region in Fig. 4, which is preferred, at $1 \sigma$, by the recent LHC searches. However, it is obvious from Fig. 4 and the values of $\Omega_{\tilde{\chi}} h^{2}$ in the table included in this figure that Eqs. (2.5) and (2.11) are incompatible even in our model.

Departure from $\Delta_{\tilde{\tau}_{2}}=0$ is not expected to alter drastically our predictions as regards the value of $m_{h}$ since $m_{h}$ depends crucially on $M_{1 / 2}$, but only mildly on $m_{0}$. Moreover, one can deduce from the slop of the left boundary of the gray regions in Fig. 2 that, increasing $\Delta_{\tilde{\tau}_{2}}$, smaller $M_{1 / 2}$ 's and, therefore, slightly lighter Higgs masses are permitted by Eq. (2.7). Note that, since $\tilde{\chi}$ cannot be the LSP, both signs of $\Delta_{\tilde{\tau}_{2}}$ are possible. In particular $\Delta_{\tilde{\tau}_{2}}>0\left[\Delta_{\tilde{\tau}_{2}}<0\right]$ corresponds to $\tilde{\chi}\left[\tilde{\tau}_{2}\right]$ being the LOSP. The critical case $\Delta_{\tilde{\tau}_{2}} \simeq 0$ gives the minimal possible relic abundance of the LOSP in both cases due to the coannihilation effect and, therefore, the maximal possible mass of the LSP if this is produced mainly non-thermally - see Refs. [40, 41, 42].

Having in mind mostly the latter possibility, we proceed in the presentation of our predictions for the sparticle and the Higgs boson spectrum of our model, which may be observable at the LHC. In Table 1, we list the model input and output parameters, the masses in $\mathrm{GeV}$ of the sparticles neutralinos $\tilde{\chi}, \tilde{\chi}_{2}^{0}, \tilde{\chi}_{3}^{0}, \tilde{\chi}_{4}^{0}$, charginos $\tilde{\chi}_{1}^{ \pm}, \tilde{\chi}_{2}^{ \pm}$, gluinos $\tilde{g}$, squarks $\tilde{t}_{1}, \tilde{t}_{2}, \tilde{b}_{1}, \tilde{b}_{2}, \tilde{u}_{L}, \tilde{u}_{R}, \tilde{d}_{L}, \tilde{d}_{R}$, and sleptons $\tilde{\tau}_{1}, \tilde{\tau}_{2}, \tilde{v}_{\tau}, \tilde{e}_{L}, \tilde{e}_{R}, \tilde{v}_{e}$ - and the Higgs bosons $\left(h, H, H^{ \pm}, A\right)$, and the values of the various low energy observables for $\Delta_{\tilde{\tau}_{2}} \simeq 0, A_{0} / M_{1 / 2}=-3,-2,-1,0$, and 2 and for the minimal $M_{1 / 2}$ allowed by Eq. (2.7) in each case. We consider the squarks and sleptons of the two first generations as degenerate. From the values of the various observable quantities, we see that the bound in Eq. (2.5) and the optional lower bound in Eq. (2.9) are violated. So, the lightest neutralino cannot be the LSP. It is also very interesting to observe that the predicted values of $m_{h}$ lie close or even inside the range in Eq. (2.11) favored by [25] - cf. Refs. [54, 57]. 
The deviation from YU can be estimated by defining [19] the relative splittings $\delta h_{b}$ and $\delta h_{\tau}$ at $M_{\mathrm{GUT}}$ through the relations:

$$
\delta h_{b} \equiv \frac{h_{b}-h_{t}}{h_{t}}=-\frac{2 c}{1+c}=-\delta h_{\tau} \equiv \frac{h_{t}-h_{\tau}}{h_{t}}
$$

Along the dotted line of Fig. 4, the ranges of the parameters $c, \delta h_{\tau}, \delta h_{b}$, and $\tan \beta$ are

$$
0.148 \lesssim c \lesssim 0.16, \quad 0.26 \lesssim \delta h_{\tau}=-\delta h_{b} \lesssim 0.28, \quad 56.2 \lesssim \tan \beta \lesssim 56.9
$$

Let us underline that, although the required deviation from YU is not so small, the restrictions from YU are not completely lost since $\tan \beta$ remains large - close to 60 - and that the deviation from exact YU is generated within well-motivated SUSY GUTs described in [16].

It is worth emphasizing that our results do not invalidate the $\tilde{\chi}$ candidacy for a CDM particle in all versions of the CMSSM with Yukawa quasi-unification. This is because the (monoparametric) condition of Eq. (??), which we considered here, is only a simplified case of the Yukawa quasi-unification conditions shown in Eq. (15) of [16], which depend on one real and two complex parameters. Actually, the investigation of the viability of $\tilde{\chi}$ as a CDM candidate within the CMSSM with Yukawa quasi-unification conditions more complicated than the one in Eq. (??) derived from the GUT models of [16] is under consideration. Alternatively, our present model may be perfectly consistent with data if we avoid the restriction from Eq. (2.5) by assuming that the LSP is the axino $[41,42]$ with mass a little lower than $m_{\tilde{\chi}}$ and that the reheat temperature is adequately low.

\section{Conclusions and further study}

We performed a revised scan of the parameter space of the CMSSM with $\mu>0$ applying a suitable Yukawa quasi-unification condition predicted by the SUSY GUT model of Ref. [16], which has been constructed in order to remedy the $b$-quark mass problem arising from exact YU and universal boundary conditions. We took into account updated constraints from collider and cosmological data. Although the neutralino-stau coannihilations drastically reduce the neutralino relic abundance and, thus, enhance the upper bound on $m_{\tilde{\chi}}$ implied by the assumption that the neutralino is a CDM particle, they do not quite succeed to bring it to an acceptable level compatible with the lower bound on $m_{\tilde{\chi}}$ induced by $B_{s} \rightarrow \mu^{+} \mu^{-}$. Therefore, - contrary to our findings in [22] $\tilde{\chi}$ is excluded as CDM particle by the combination of the constraints from $B_{s} \rightarrow \mu^{+} \mu^{-}$and CDM. As a consequence, the model can become consistent with observations only if the LSP is a SUSY particle other than the neutralino. This could be the axino or the gravitino and can account for the present $\mathrm{CDM}$ abundance in the universe. It is interesting to note that, in this case, the lowest predicted $m_{h}$ is enhanced and gets closer to the range favored by the recent preliminarily results announced by LHC.

Further progress is being made towards the modification of the model, in order for the neutralino to be the LSP. We are studying a more general form of the model, where we have $S U(2)_{R}$ triplets along with the $S U(2)_{R}$ singlets and lower $\tan \beta$ values, but still keep the 'quasi-unification' of the Yukawa couplings. 
Table 1: Input and output parameters, masses of the sparticles and Higgs bosons, and values of the low energy observables for $\Delta_{\tilde{\tau}_{2}} \simeq 0$, five values of $A_{0} / M_{1 / 2}$, and the minimal $M_{1 / 2}$.

\begin{tabular}{|c|c|c|c|c|c|}
\hline \multicolumn{6}{|c|}{ Input parameters } \\
\hline$A_{0} / M_{1 / 2}$ & -3 & -2 & -1 & 0 & 2 \\
\hline$c$ & 0.1589 & 0.1592 & 0.1585 & 0.153 & 0.1475 \\
\hline$M_{1 / 2} / \mathrm{GeV}$ & 2355.02 & 2019.6 & 1744.65 & 1530.3 & 1317.65 \\
\hline$m_{0} / \mathrm{GeV}$ & 4292.06 & 2760.01 & 1691.01 & 1132.04 & 1542.88 \\
\hline \multicolumn{6}{|c|}{ Output parameters } \\
\hline $\tan \beta$ & 57.1 & 56.9 & 56.3 & 56.2 & 56.2 \\
\hline $100 \delta h_{\tau}\left(M_{\mathrm{GUT}}\right)$ & 27.4 & 27.4 & 27.3 & 26.5 & 25.7 \\
\hline$\mu / \mathrm{GeV}$ & 3704.64 & 2755 & 2059 & 1588 & 1250 \\
\hline \multicolumn{6}{|c|}{ Masses in $\mathrm{GeV}$ of sparticles and Higgs bosons } \\
\hline$\tilde{\chi}$ & 1089.2 & 926.5 & 794.2 & 692.5 & 595.2 \\
\hline$\tilde{\chi}_{2}^{0}$ & 2087.2 & 1777.7 & 1524.2 & 1325.4 & 1130.1 \\
\hline$\tilde{\chi}_{3}^{0}$ & 3688.5 & 2747.6 & 2057.3 & 1588.9 & 1254.8 \\
\hline$\tilde{\chi}_{4}^{0}$ & 3789.7 & 2750.3 & 2062.7 & 1600.5 & 1279.9 \\
\hline$\tilde{\chi}_{1}^{ \pm}$ & 3690.1 & 2750.6 & 2062.8 & 1600.4 & 1279.6 \\
\hline$\tilde{\chi}_{2}^{ \pm}$ & 2087.3 & 1777.8 & 1524.3 & 1325.5 & 1130.2 \\
\hline$\tilde{g}$ & 5190.9 & 4454 & 5190.9 & 3388.6 & 2981.2 \\
\hline$\tilde{t}_{1}$ & 4336.3 & 3608.2 & 3094.3 & 2752.2 & 2567.8 \\
\hline$\tilde{t}_{2}$ & 3593.2 & 3084.5 & 2709.5 & 2449.2 & 2303.1 \\
\hline$\tilde{b}_{1}$ & 4514.1 & 3653.4 & 3097.4 & 2747.9 & 2575.7 \\
\hline$\tilde{b}_{2}$ & 4310.6 & 3561.2 & 3005.7 & 2644.1 & 2508.8 \\
\hline$\tilde{u}_{L}$ & 6215.9 & 4786 & 3815.1 & 3231.7 & 3051.1 \\
\hline$\tilde{u}_{R}$ & 6047.6 & 4624.8 & 3661.4 & 3090.5 & 2941.3 \\
\hline$\tilde{d}_{L}$ & 6216.3 & 4786.5 & 3815.8 & 3232.5 & 3052 \\
\hline$\tilde{d}_{R}$ & 6026.1 & 4604.4 & 3641.9 & 3072.7 & 2927.7 \\
\hline$\tilde{\tau}_{1}$ & 3447.3 & 2413.2 & 1721.0 & 1354.4 & 1436.4 \\
\hline$\tilde{\tau}_{2}$ & 1089.9 & 927.1 & 794.4 & 692.5 & 595.7 \\
\hline$\tilde{v}_{\tau}$ & 3443.9 & 2407.7 & 1712.4 & 1343.3 & 1430.2 \\
\hline$\tilde{e}_{L}$ & 4582.6 & 3085.1 & 2070.4 & 1544.9 & 1789.6 \\
\hline$\tilde{e}_{R}$ & 4389.7 & 2869.5 & 1821.4 & 1278.5 & 1626.4 \\
\hline$\tilde{v}_{e}$ & 4581.6 & 3083.7 & 2068.6 & 1542.6 & 1787.5 \\
\hline$h$ & 126.17 & 124.3 & 122.68 & 121.15 & 119.30 \\
\hline$H$ & 1463.72 & 1334.6 & 1181.53 & 1012.4 & 730.78 \\
\hline$H^{ \pm}$ & 1466.38 & 1337.6 & 1185.04 & 1016.55 & 736.91 \\
\hline$A$ & 1463.99 & 1334.9 & 1182.00 & 1013 & 732 \\
\hline \multicolumn{6}{|c|}{ Low energy observables } \\
\hline $10^{4} b \rightarrow s \gamma$ & 3.25 & 3.23 & 3.22 & 3.23 & 3.35 \\
\hline $10^{8} B_{s} \rightarrow \mu^{+} \mu^{-}$ & 1.08 & 1.08 & 1.08 & 1.08 & 1.08 \\
\hline$B_{u} \rightarrow \tau \nu$ & 0.929 & 0.915 & 0.893 & 0.856 & 0.736 \\
\hline $10^{10} \delta a_{\mu}$ & 0.565 & 1.09 & 2.04 & 3.27 & 3.4 \\
\hline$\Omega_{\tilde{\chi}} h^{2}$ & 0.301 & 0.219 & 0.167 & 0.152 & 0.151 \\
\hline
\end{tabular}




\section{References}

[1] A.H. Chamseddine, R.L. Arnowitt, and P. Nath, Phys. Rev. Lett. 49, 970 (1982); P. Nath, R.L. Arnowitt, and A.H. Chamseddine, Nucl. Phys. B227, 121 (1983); L.J. Hall, J.D. Lykken, and S. Weinberg, Phys. Rev. D 27, 2359 (1983).

[2] R. Arnowitt and P. Nath, Phys. Rev. Lett. 69, 725 (1992); G.G. Ross and R.G. Roberts, Nucl. Phys. B377, 571 (1992); V.D. Barger, M.S. Berger, and P. Ohmann, Phys. Rev. D 49, 4908 (1994); G.L. Kane, C.F. Kolda, L. Roszkowski, and J.D. Wells, ibid. 49, 6173 (1994).

[3] J.R. Ellis, T. Falk, and K.A. Olive, Phys. Lett. B 444, 367 (1998); J.R. Ellis, T. Falk, K.A. Olive, and M. Srednicki, Astropart. Phys. 13, 181 (2000); 15, 413(E) (2001).

[4] A.B. Lahanas, D.V. Nanopoulos, and V.C. Spanos, Phys. Rev. D 62, 023515 (2000); J.R. Ellis, T. Falk, G. Ganis, K.A. Olive, and M. Srednicki, Phys. Lett. B 510, 236 (2001).

[5] G. Lazarides and C. Panagiotakopoulos, Phys. Lett. B 337, 90 (1994); S. Khalil, G. Lazarides, and C. Pallis, ibid. 508, 327 (2001).

[6] B. Ananthanarayan, G. Lazarides, and Q. Shafi, Phys. Rev. D 44, 1613 (1991); Phys. Lett. B 300, 245 (1993).

[7] I. Antoniadis and G.K. Leontaris, Phys. Lett. B 216, 333 (1989).

[8] R. Jeannerot, S. Khalil, G. Lazarides, and Q. Shafi, J. High Energy Phys. 10, 012 (2000).

[9] H. Baer, M.A. Diaz, J. Ferrandis, and X. Tata, Phys. Rev. D 61, 111701 (2000); H. Baer, M. Brhlik, M.A. Diaz, J. Ferrandis, P. Mercadante, P. Quintana, and X. Tata, ibid. 63, 015007 (2000); D. Auto, H. Baer, C. Balázs, A. Belyaev, J. Ferrandis, and X. Tata, J. High Energy Phys. 06, 023 (2003); D. Auto, H. Baer, A. Belyaev, and T. Krupovnickas, ibid. 10, 066 (2004).

[10] T. Blažek, R. Dermíšek, and S. Raby, Phys. Rev. Lett. 88, 111804 (2002); Phys. Rev. D 65, 115004 (2002); R. Dermíšek, S. Raby, L. Roszkowski, and R. Ruiz de Austri, J. High Energy Phys. 04, 037 (2003).

[11] M.S. Carena, M. Olechowski, S. Pokorski, and C.E.M. Wagner, Nucl. Phys. B426, 269 (1994); R. Hempfling, Phys. Rev. D 49, 6168 (1994); L.J. Hall, R. Rattazzi, and U. Sarid, ibid. 50, 7048 (1994).

[12] D.M. Pierce, J.A. Bagger, K.T. Matchev, and R. Zhang, Nucl. Phys. B491, 3 (1997); M.S. Carena, D. Garcia, U. Nierste, and C.E.M. Wagner, ibid. B577, 88 (2000).

[13] P.Z. Skands et al., J. High Energy Phys. 07, 036 (2004).

[14] S.F. King and M. Oliveira, Phys. Rev. D 63, 015010 (2001); I. Gogoladze, R. Khalid, and Q. Shafi, ibid. 79, 115004 (2009); 80, 095016 (2009); I. Gogoladze, R. Khalid, S. Raza, and Q. Shafi, J. High Energy Phys. 12, 055 (2010), I. Gogoladze, R. Khalid, S. Raja and Q. Shafi, J. High Energy Phys. 06, 117 (2011), I. Gogoladze, Q. Shafi and C.S. Un, arXiv:1112.2206, I. Gogoladze, Q. Shafi and C.S. Un, arXiv:1203.6082.

[15] U. Chattopadhyay and P. Nath, Phys. Rev. D 65, 075009 (2002); U. Chattopadhyay, A. Corsetti, and P. Nath, ibid. 66, 035003 (2002); C. Pallis, Nucl. Phys. B678, 398 (2004).

[16] M.E. Gómez, G. Lazarides, and C. Pallis, Nucl. Phys. B638, 165 (2002).

[17] R. Jeannerot, S. Khalil, and G. Lazarides, J. High Energy Phys. 07, 069 (2002); G. Lazarides and A. Vamvasakis, Phys. Rev. D 76, 083507 (2007); 76, 123514 (2007); G. Lazarides, I.N.R. Peddie, and A. Vamvasakis, ibid. 78, 043518 (2008); G. Lazarides, arXiv:1006.3636. 
[18] M.E. Gómez, G. Lazarides, and C. Pallis, Phys. Rev. D 67, 097701 (2003).

[19] G. Lazarides and C. Pallis, hep-ph/0404266; hep-ph/0406081.

[20] M. Davier, A. Hoecker, B. Malaescu, and Z. Zhang, Eur. Phys. J. C 71, 1515 (2011).

[21] F. Jegerlehner and R. Szafron, Eur. Phys. J. C 71, 1632 (2011).

[22] N. Karagiannakis, G. Lazarides, and C. Pallis, Phys. Lett. B 704, 43 (2011).

[23] S. Dar, I. Gogoladze, Q. Shafi, and C.S. Un, Phys. Rev. D 84, 085015 (2011).

[24] CMS and LHCb Collaborations, CMS-PAS-BPH-11-019, LHCb-CONF-2011-047, http: //cdsweb.cern. ch/record/1374913/files/BPH-11-019-pas.pdf.

[25] Update on the Standard Model Higgs searches in ATLAS and CMS, talks by F. Gianotti and G. Tonelli, 13/12/2011, CERN, http: //indico.cern.ch/conferenceDisplay.py? confld=164890.

[26] B.C. Allanach, Computer Physics Commun. 143, 305 (2002).

[27] G. Belanger, F. Boudjema, A. Pukhov, and A. Semenov, http: / / lapth.in2p3.fr/micromegas; G. Belanger, F. Boudjema, P. Brun, A. Pukhov, S. Rosier-Lees, P. Salati, and A. Semenov, Comput. Phys. Commun. 182, 842 (2011).

[28] R. Trotta, F. Feroz, M.P. Hobson, L. Roszkowski, and R. Ruiz de Austri, J. High Energy Phys. 12, 024 (2008); A. Belyaev, S. Dar, I. Gogoladze, A. Mustafayev, and Q. Shafi, arXiv:0712.1049 [hep-ph]; O. Buchmueller et al., Eur. Phys. J. C 64, 391 (2009); Y. Akrami, P. Scott, J. Edsjo, J. Conrad, and L. Bergstrom, J. High Energy Phys. 04, 057 (2010); L. Roszkowski, R. Ruiz de Austri, and R. Trotta, Phys. Rev. D 82, 055003 (2010); O. Buchmueller et al., arXiv:1110.3568.

[29] H. Baer, S. Kraml, S. Sekmen, and H. Summy, J. High Energy Phys. 03, 056 (2008); 10, 079 (2008); H. Baer, S. Kraml, and S. Sekmen, ibid. 09, 005 (2009); H. Baer, S. Kraml, A. Lessa, and S. Sekmen, ibid. 02, 055 (2010); H. Baer, S. Kraml, A. Lessa, S. Sekmen, and H. Summy, Phys. Lett. B 685, 72 (2010).

[30] K. Nakamura et al. [Particle Data Group], J. Phys. G 37, 075021 (2010).

[31] H. Baer, J. Ferrandis, K. Melnikov, and X. Tata, Phys. Rev. D 66, 074007 (2002); K. Tobe and J.D. Wells, Nucl. Phys. B663, 123 (2003).

[32] Tevatron Electroweak Working Group [CDF and D0 collaborations], arXiv:0903.2503; T. Aaltonen et al. [CDF Collaboration], Phys. Rev. Lett. 105, 252001 (2010).

[33] E. Komatsu et al. [WMAP Collaboration], Astrophys. J. Suppl. 192, 18 (2011); http://lambda.gsfc.nasa.gov/product/map.

[34] M.E. Gómez, G. Lazarides, and C. Pallis, Phys. Rev. D 61, 123512 (2000); Phys. Lett. B 487, 313 (2000).

[35] T. Nihei, L. Roszkowski, and R. Ruiz de Austri, J. High Energy Phys. 05, 063 (2001); ibid. 07, 024 (2002).

[36] G. Bélanger, F. Boudjema, A. Pukhov, and A. Semenov, Comput. Phys. Commun. 174, 577 (2006).

[37] G.F. Giudice, E.W. Kolb, and A. Riotto, Phys. Rev. D 64, 023508 (2001); N. Fornengo, A. Riotto, and S. Scopel, ibid. 67, 023514 (2003); G.B. Gelmini and P. Gondolo, ibid. 74, 023510 (2006); G. Barenboim and J.D. Lykken, J. High Energy Phys. 12, 005 (2006); A.B. Lahanas, N.E. Mavromatos and D.V. Nanopoulos, Phys. Lett. B 649, 83 (2007); M. Drees, H. Iminniyaz, and M. Kakizaki, Phys. Rev. D 76, 103524 (2007). 
[38] C. Pallis, Astropart. Phys. 21, 689 (2004); J. Cosmol. Astropart. Phys. 10, 015 (2005); Nucl. Phys. B751, 129 (2006); hep-ph/0610433.

[39] K. Matchev, hep-ph/0402088; E.A. Baltz, astro-ph/0412170; G. Lazarides, hep-ph/0601016; M. Taoso, G. Bertone, and A. Masiero, J. Cosmol. Astropart. Phys. 03, 022 (2008).

[40] J.R. Ellis, K.A. Olive, Y. Santoso, and V.C. Spanos, Phys. Lett. B 588, 7 (2004); K. Jedamzik, K.-Y. Choi, L. Roszkowski, and R. Ruiz de Austri, J. Cosmol. Astropart. Phys. 07, 007 (2006); J. Pradler and F.D. Steffen, Phys. Lett. B 666, 181 (2008).

[41] L. Covi, L. Roszkowski, R. Ruiz de Austri, and M. Small, J. High Energy Phys. 06, 003 (2004); K.-Y. Choi, L. Covi, J.E. Kim, and L. Roszkowski, arXiv:1108.2282.

[42] H. Baer and H. Summy, Phys. Lett. B 666, 5 (2008); H. Baer, M. Haider, S. Kraml, S. Sekmen, and H. Summy, J. Cosmol. Astropart. Phys. 02, 002 (2009); H. Baer, A.D. Box, and H. Summy, J. High Energy Phys. 10, 023 (2010).

[43] E. Barberio et al. [Heavy Flavor Averaging Group], arXiv:0808.1297.

[44] F. Borzumati and C. Greub, Phys. Rev. D 58, 074004 (1998); M. Misiak et al., Phys. Rev. Lett. 98, 022002 (2007)

[45] M. Ciuchini, G. Degrassi, P. Gambino, and G.F. Giudice, Nucl. Phys. B527, 21 (1998); G. Degrassi, P. Gambino, and G.F. Giudice, J. High Energy Phys. 12, 009 (2000); M.E. Gómez, T. Ibrahim, P. Nath, and S. Skadhauge, Phys. Rev. D 74, 015015 (2006).

[46] P.H. Chankowski and L. Slawianowska, Phys. Rev. D 63, 054012 (2001); C.S. Huang, W. Liao, Q.S. Yan, and S.H. Zhu, ibid. 63, 114021 (2001); 64, 059902(E) (2001); C. Bobeth, T. Ewerth, F. Kruger, and J. Urban, ibid. 64, 074014 (2001); A. Dedes, H.K. Dreiner, and U. Nierste, Phys. Rev. Lett. 87, 251804 (2001); J.R. Ellis, K.A. Olive, and V.C. Spanos, Phys. Lett. B 624, 47 (2005).

[47] F. Mahmoudi, Comput. Phys. Commun. 180, 1579 (2009).

[48] T. Aaltonen et al. [CDF Collaboration], Phys. Rev. Lett. 100, 101802 (2008).

[49] G. Isidori and P. Paradisi, Phys. Lett. B 639, 499 (2006); G. Isidori, F. Mescia, P. Paradisi, and D. Temes, Phys. Rev. D 75, 115019 (2007).

[50] S.P. Martin and J.D. Wells, Phys. Rev. D 64, 035003 (2001).

[51] G.W. Bennett et al. [Muon $g$ - 2 Collaboration], Phys. Rev. D 73, 072003 (2006).

[52] K. Hagiwara, R. Liao, A.D. Martin, D. Nomura, and T. Teubner, J. Phys. G 38, 085003 (2011).

[53] The LEP Collaborations ALEPH, DELPHI, L3, OPAL, The LEP Working Group for Higgs Boson Searches, Eur. Phys. J. C 47, 547 (2006).

[54] O. Buchmueller et al., arXiv:1112.3564.

[55] G. Degrassi, P. Slavich, and F. Zwirner, Nucl. Phys. B611, 403 (2001); A. Brignole, G. Degrassi, P. Slavich, and F. Zwirner, ibid. B631, 195 (2002); ibid. B643, 79 (2002); A. Dedes, G. Degrassi, and P. Slavich, ibid. B672, 144 (2003).

[56] B.C. Allanach, S. Kraml, and W. Porod, J. High Energy Phys. 03, 045 (2003); B.C. Allanach, A. Djouadi, J.L. Kneur, W. Porod, and P. Slavich, ibid. 09, 044 (2004).

[57] D. Albornoz Vasquez, G. Belanger, R.M. Godbole, and A. Pukhov, arXiv:1112.2200; I. Gogoladze, Q. Shafi, and C.S. Un, arXiv:1112.2206. 